\title{
Prospects of Some Applications of Engineered Nanomaterials: A review
}

\author{
Ibtisam Kamal* \\ Faculty of Science, Soran University, Iraq \\ Received: 眥 October 17, 2018; Published: 些 October 24, 2018 \\ *Corresponding author: Ibtisam Kamal, Faculty of Science, Soran University, Iraq
}

\begin{abstract}
Nanomaterials are materials engineered with extremely small particle size having at least one dimension in the range of 1 to 100 nanometers $(\mathrm{nm})$. It is well established that when materials are produced in nano scale, they demonstrate unique electronic, magnetic, catalytical as well as optical properties that candidate them for wide range of applications in engineering, medical and environmental fields. An extensive variety of nanomaterials has been manufactured, and already been used in many products and much more extensive utilization is expected in the future. The contribution of nanotechnology to research and development in industrial sectors results in increasing the efficiency and productivity of the industrial processes and brings huge promise in producing new nanomaterials and products, with tailored characteristics that offer unique properties compared to the bulk materials. The present review elucidates the role of nanomaterials and its applications in coatings, sensors, adsorbents, drug delivery, and catalysts. Several research articles that are addressing the applications of nanomaterials in these fields are included. Limitations hinder the large scale production and applications of nanomaterials and the future prospects are also summarized.
\end{abstract}

Keywords: Nano-Materials; Applications; Coatings; Sensors; Adsorbents; Drug Delivery; Catalysts

\section{Introduction}

It is well known that physics, mathematics, biology, computer, chemistry, material, medicine and engineering sciences, have all vital roles in the development and manufacturing of novel nanomaterials with tailored properties. Nanomaterials are synthesized for largescale by the top-down and bottom-up production approaches [1]. The miniaturization of materials using these two approaches resulted in fabrication of the one-dimensional nanomaterials, such as thin films and engineered surfaces, two dimensional such as nanotubes and nanowires, and three dimensional, like multilayer and fibrous nanomaterials. All these classes of nanomaterials generated immense interest among the scientific community in recent years due to their unique properties, mainly arising from the quantum confinement effect and extremely large surface-tovolume ratios, and many of them already are being commercialized and used in various applications [2].

Nanotechnology products maturity could be categorized to products in pilot testing, batch production and bulk production [3]. Scaling up the production of nanomaterials is time, cost and efforts intensive process. Laboratory experiments are usually focused on production sub-gram quantities of the nanomaterial after several steps including the selection the appropriate raw materials and reagents, optimization the methods and establishing the optimal conditions of preparation, understanding the formation mechanisms, characterization the products and studying their properties, and exploring and approving the relevant applications. Furthermore, the research efforts is usually turned in the development of novel environmentally friendly and economical production methods in order to make their production more attractive to the industrial sector [4].

Scaling up the process to industrial scale needs to develop an economical mass-production method by exploring the efficient design of the equipment and manufacturing processes alongside with cost estimation studies. Although numerous numbers of nanomaterials are commercially manufactured, the industrial use of nanomaterials faces many obstacles owing to the lack of suitable regulatory framework and guidance on safety requirements, as a result lot of promising new nanomaterials never reach the market. The present review highlights the applications of some nanomaterials in the fields of thin coatings, sensors, adsorbents, drug delivery, and catalysts. Limitations hinder the large scale production and applications are also included. 


\section{Some Possible Applications of Nanomaterials}

\section{Thin coatings field}

A coating material is a material in liquid, paste or powder form which, when applied, forms a coat on the material surface. Surfaces are coated to prevent contact with severe environments and therefore protect the underlying surface under the condition of reserving its original properties. The application of thin films and engineered surfaces have been developed and used for decades in fields of electronic devices in particular magnetic storage media and semiconductor devices, as well as in chemistry and engineering fields. The efficient using of these materials is able to open up exciting opportunities for protection and multifunctional modification of the surface properties. Nanomaterials possess high specific surface areas. In coatings, "surface" translates into "interface" between particle and medium. A higher proportion of interfaces results in more interaction between matrix and particle and thus a toughening of the material. Also, the use of small particles permits the application of thinner coatings, or simply the use of less coating material [5]. In addition, many studies have proven that coating with nanomaterials resulted in more compact and less porosity coating compared to conventional coating [6]. Nanotechnologybased functional coatings typically contain titanium dioxide, silicon dioxide, carbon black, iron oxide, zinc oxide and silver.

Regarding the applications of nanomaterials in the field of thin coating, various stable nanomaterials based thin films coatings were synthesized using wet-chemical approach, electrodeposition method, and microwave irradiation technique. The fabricated thin film devices have been investigated and suggested for promising applications in different industries. Herein, are several examples of nanomaterials that can be used for thin film coatings. Nanographenes have been tested as protective layers for molecular electronics [7]. Coated $\mathrm{Cu}_{2} \mathrm{ZnSnS}_{4}$ nanoparticles were used as coating films for solar cell applications [8]. ZnO nanoparticle based thin-film were prepared and analyzed as transistors for flexible electronic applications [9]. Silver nanofilm deposited on glass chips are used as Surface Enhanced Raman scattering (SERS) substrates for detection and monitoring of permethrin in environment and on foods [10]. Titanium dioxide $\left(\mathrm{TiO}_{2}\right)$ nanoparticles of high photocatalytic activity and antibacterial properties were prepared by sol-gel process and used as thin layers for self- cleaning transparent coatings for windows in outdoors applications [11].

The influence of silica $\left(\mathrm{SiO}_{2}\right)$ nanoparticle incorporation in a sol-gel based coating was studied. The $\mathrm{SiO}_{2}$ particle surface treatment improved coating homogeneity and prevented cracking propagation and the corrosion resistance improved with functionalized $\mathrm{SiO}_{2}$ nanoparticles [12]. It has been reported that creation of nanoscale binary composition film composed of $\mathrm{SiO}_{2}$ nanoparticles and appropriate polymer allowed a super hydrophobic property which assure maximum protection against water of stone based monuments [13]. A new nanocomposite films of diamond-like carbon with silver nanoparticle DLC: Ag were deposited on textile (synthetic silk) as a part of a smart bandage prototype demonstrating antibacterial properties. The DLC:Ag layer was stabilized on the surface of the bandage using a thin layer of medical grade gelatin and cellulose [14]. The bandage structure is shown in Figure 1. Recently, polymer coated metal/ metal oxide-carbon nanotubes coated with a thin polymeric film of polydopamine were successfully synthesized and used to fabricate thin film nanocomposite membranes for water desalination applications [15]. A film of Silver (Ag) nanoparticles in a $\mathrm{ZnO}$ matrix of tunable optical properties that candidate its usefulness for plasmonic applications was successfully synthesized by RFmagnetron sputtering at room temperature [16].

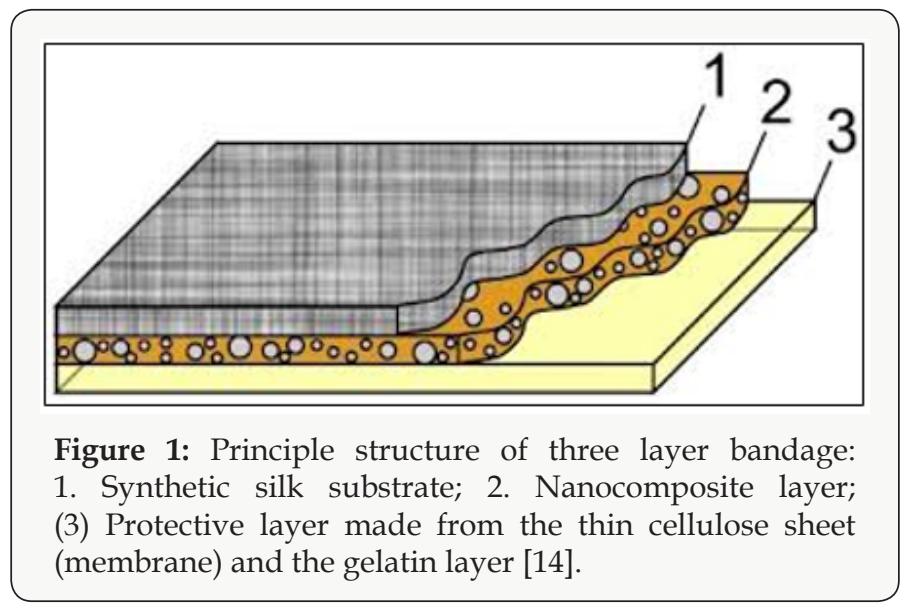

\section{Sensing field}

Nanosensors are sensors whose active elements include nanomaterials. The nanostructures (size) and the materials being used are essential in developing nanosensors such as: nanoscale wires (capability of extreme detection sensitivity), carbon nanotubes (very high surface area), thin films, and nanoparticles. Nanosensors are categorized to different classes including and not limited to chemical nanosensors (e.g., detecting very small amounts of chemical vapors), nanobiosensors (e.g., cancer detection) and nanoscale electrometers [17]. Carbon-based nanomaterials have wide range of applications including detecting heavy metal ions, gas molecules, food additives, antibodies, and toxic pesticides, as well as reporters for bioimaging [18]. On the other hand, the unique physicochemical, spectral and optical properties of noble metal nanoparticles have allowed for the development of new biosensors [19]. The use of gold NPs and its functionalization as efficient sensors in colorimetric detection of toxic metal ions has been reviewed [20]. A quantitative, sensitive and naked eye colorimetric sensing of bivalent copper ions using bio-inspired synthesis of size and shape controlled silver nanocapsules (AgNCs) is reported for selectively and sensitively the $\mathrm{Cu}^{2+}$ ion in industrial effluents with an ultralow detection limit of $2.6 \times 10-9$ mol L-1 [21]. Figure 2 illustrates the sensing route of silver nanocapsules. 


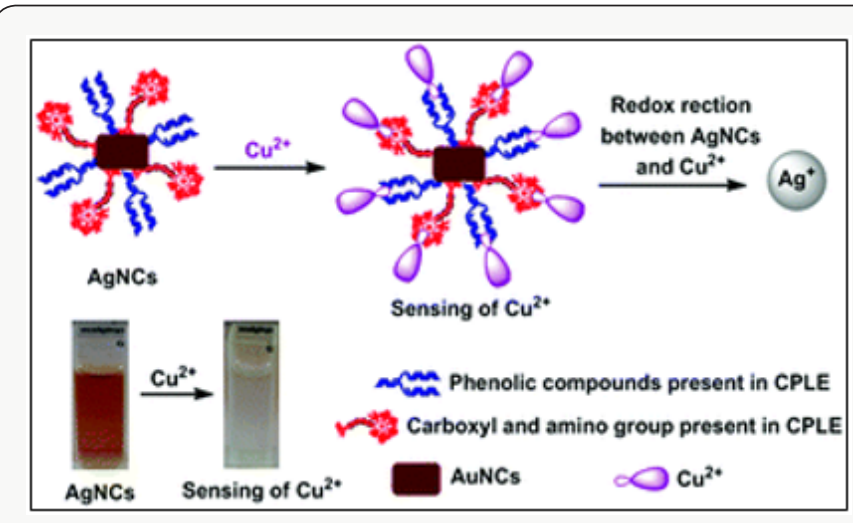

Figure 2: Sensing Copper ions by silver nanocapsules [21].

Pd-NPs doped with chitosan-graphene have been employed as biosensors for glucose [22]. Metal oxide nanowires are found to be promising class of sensing materials owing to easy fabrication methods and chemical stability. ZnO nanowires synthesized via chemical vapor deposition showed promising suitability for sensing applications [23]. Boron-doped silicon nanowires were used to create highly sensitive, real-time electrically based sensors for biological and chemical species [24]. There are excellent reviews available on nano sensors, among is a review on the innovations and challenges in the field of metal oxide nanowires chemical sensors [25]. The synthetic strategies and the applications of Carbon Nanotubes Hybrid Assemblies in amperometric sensors, as well as the mechanisms, principles and the performances of chemical sensors and biosensors are comprehensively reviewed elsewhere [26].

\section{Adsorption field}

As known, the growing urbanization and industrialization of human civilization result in discharge of huge amount of hazardous pollutants into air, soil and aquatic streams. The hazardous contaminants produce detrimental impact on the biotic resources [27]. The huge developments in nanoscience and nanotechnology has shown interesting potential for producing nanostructure adsorbents that exhibited higher efficiency and faster removal rate of pollutants compared to the conventional adsorbents. A variety of efficient, cost-effective and eco- friendly nanomaterials with unique functionalities have been developed for remediation of industrial effluents, groundwater, surface water and drinking water [28-30] including nanoadsorbents and nanostructured membranes as substituents to the current remediation agents.

In this regard, there are several examples of nanoparticles and nanomaterials that can be used for remediation of water, e.g. iron-based nanoparticles showed a very high rate of adsorption of pollutants from drinking water [31]. Magnetic nanoparticles are among the most important group of nanoparticles used as adsorbents. Magnetite $\left(\mathrm{Fe}_{3} \mathrm{O}_{4}\right)$ nanoparticles showed preferential reactivity for trace elements removal and/or immobilization trace elements from contaminated waters and/or soils [32]. They are also used for water purifications and degradation of organic compounds [33]. A magnetic hetero structured adsorbent: zinc ferrite $\left(\mathrm{ZnFe}_{2} \mathrm{O}_{4}\right)$ /organoclay nanocomposite showed efficient performance for water purification from some dye pollutants [34]. Figure 3 shows the adsorption of dye pollutants onto the prepared nanocomposite. Copper oxide nano-particles were prepared and fully characterized considering their adsorption properties of heavy metals. It was proved that they are promising adsorbent for the removal of heavy metals from aqueous solutions [30]. Nickel based nanoparticles were also proved as efficient adsorbents for water purifications [35]. Recently, new generation of nanoadsorbents were prepared and tested for removal of water contamination due to the drugs and pharmaceuticals residues. They were found to be capable to remove the pollutants even at low concentration i.e. $\mu \mathrm{g} / \mathrm{L}$ under varied conditions of $\mathrm{pH}$ and temperature [36]. The use of nanomaterials for the remediation of different environmental contaminants is highlighted in a new review by [37].

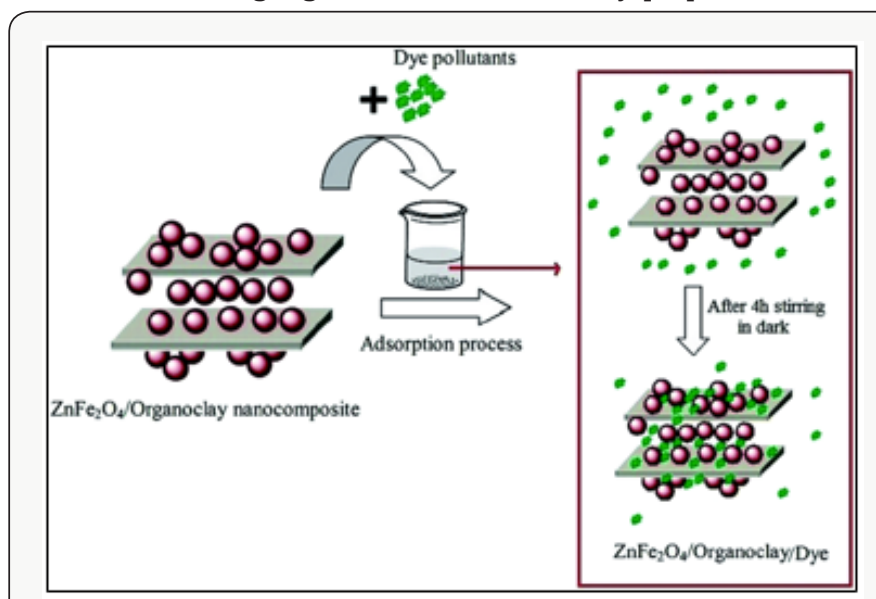

Figure 3: Schematic diagram of adsorption of dye pollutants onto a nanocomposite [34].

On the other hand, the enhanced properties and effectiveness of nanotechnology-based materials makes them particularly suitable for removing contaminants from air and industrial gases. Nanocrystalline $\mathrm{MgO}, \mathrm{CaO}$ and $\mathrm{Al}_{2} \mathrm{O}_{3}$ have shown remarkably high capacities to chemically adsorb typical volatile organic compounds that are representative of air pollutants [38]. It is worthy to note that $\mathrm{CO}_{2}$ and $\mathrm{CH}_{4}$ are the two most important global warming gases. The $\mathrm{Fe}_{3} \mathrm{O}_{4}$-graphene based nanoparticles are among the nanomaterials used to capture these gases due to their high thermal stability and high porosity [39]. The application of nano-molybdenum oxide adsorbent in natural gas sweetening process (removal of $\mathrm{H}_{2} \mathrm{~S}$ ) has been demonstrated [40]. A comparative study has been performed on using carbon nanomaterials for $\mathrm{SO}_{2}$ gas removing by adsorption [41]. An interesting review on the application of nanotechnology for air pollution control is presented elsewhere [42].

\section{Drug Delivery Field}

The use of nanotechnology in medical field and more specifically drug delivery is growing rapidly. The aims for nanoparticle 
entrapment of drugs are enhanced delivery to, or uptake by target cells and/or a reduction in the toxicity of the free drug to non-target organs. Drugs can be either encapsulated within the nanoparticle or attached to the surface. The main research topics related to design an appropriate nano-drug delivery system is: size, shape and surface chemistry of the nanoparticles, drug incorporation and release, its formulation stability and shelf life, its biocompatibility and biodistribution and targeting, and its functionality. In connection, biodegradable nanoparticles with a limited life span as long as therapeutically needed would be optimal. Controlling and optimization of all the aforementioned issues will result in creation of long-lived and target-specific nano-based drug delivery system [43]. For the purpose of time and resources effectiveness, the optimizing of these parameters is usually conducted through designed experiments aided with a computational modeling [44].

A variety of nanostructured drug delivery carriers have been studied including liposomes, polymers, dendrimers, silicon or carbon materials, and magnetic nanoparticles. The analysis of nanocarriers and their connections with drugs are reviewed by many authors including [45]. The recent developments in the preparation and use of nanoparticles in drug delivery are overviewed by [46]. Metal NPs synthesized for biological applications are commonly prepared using the colloidal synthesis method. This approach utilizes a metal precursor, a reductant, and a stabilizer, and allows for the facile tuning of the size, shape, and properties of the nanostructures. The surface functionalization is one of the most favorable properties of nanoparticles used in biomedical applications. The surface of nanoparticles can be functionalized with various biomolecules, such as DNA, peptides, and antibodies throughout two kinds of interactions; noncovalent and covalent. Antibiotics or other drug molecules are able to directly conjugate with nanoparticles via ionic or covalent bonding, or by physical absorption. Figure 4 shows the possible modification and functionalization of a magnetic nanoparticles.

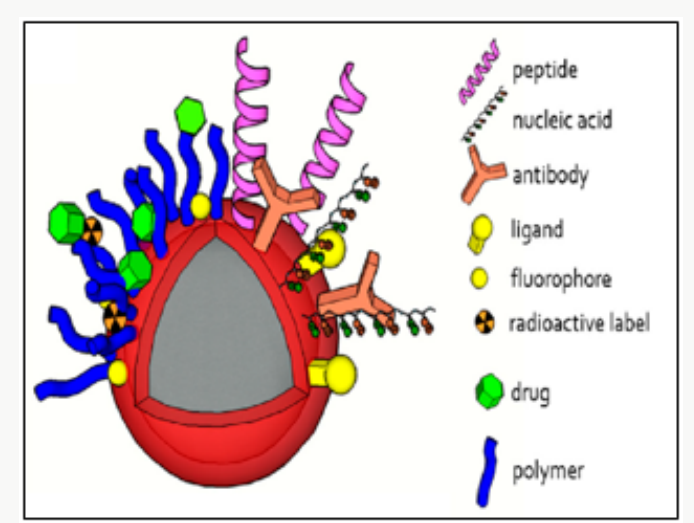

Figure 4: Possible modification and functionalization of a magnetic nanoparticles [47].

On the other hand, gene therapy presents an ideal strategy for the treatment of genetic as well as acquired diseases. It involves modification of the genetic content of the cell through the introduction of genes. This approach makes it possible to permanently correct the defective gene and thus provide a definitive cure [48]. Studies have suggested that metallic nanoparticles like $\mathrm{Au}$ and Ag, metallic oxides nanoparticles like iron oxide, and carbon nanotubes are attractive candidates of delivering all kinds of oligonucleotides such as plasmids, double stranded DNA (dsDNA), single stranded DNA (ssDNA), and single stranded RNA (ssRNA). The oligonucleotides nano delivery systems could have excellent therapeutic effects [49]. Nonviral gene delivery systems based on molecular nanoparticles with improved properties for the efficient transport of nucleic acid payloads for gene therapy was developed to mediate the safe delivery of nucleic acids to the target cells [50]. Polymeric nanoparticles including micelles/polyplexes, crosslinked micelles, polymersomes, microgels, and nanogels have been heavily investigated, they showed virus mimetic functionalities candidate them for RNA delivery [51a]. Also, polymeric nanoparticles including chitosan coated nanoparticles have been prepared and tested for protein delivery [51b, 52]. In connection, nanoparticles including gold nanoparticles have been extensively used as vaccine platforms which have potential advantages over traditional vaccine platforms, due to their varying sizes, shapes and tunable surface properties. Liu and his coworkers reviewed the use of gold nanoparticles in HIV/acquired immune deficiency [53], while, Kong and his team demonstrated the use of Au nanoparticles in vaccines delivery [54].

\section{Catalysis Field}

A catalyst is a solid phase that ideally contains large enough amounts of site for chemical reactants to adsorb, reacts, and desorbs. Chemically, catalysts are the substances that enhance the rate of a chemical reaction resulting in desired product. The catalytic power of a catalyst lies in its ability to accelerate the chemical reaction by decreasing the energy barrier i.e. activation energy (Ea) for the conversion of reactants to product (Figure 5). Catalyzed reactions often require low consumption of energy and occur at low temperature when compared to the reaction that lacks a catalyst.

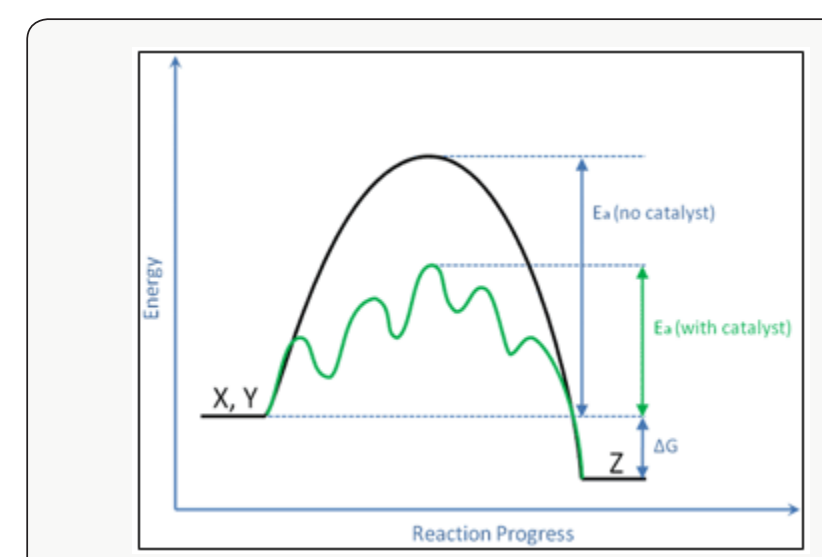

Figure 5: Potential energy diagrams for a single-step reaction in the presence and absence of a catalyst. 
The catalytic particle size must be small for expanding the surface area and increasing the numbers of catalyst sites [55]. In recent years, nanocatalysis has become an emerging field of science due to its high activity, selectivity and productivity. Nanoparticles, due to their small size exhibit high surface to volume ratio and provide larger surface area which can increase speed of chemical reaction efficiently. The structure- and shape-based properties at the nanoscale, can also affect the catalytic activity of a nanomaterial compared to bulk materials [56-58]. Nano particles have the ability to catalyze reactions by interacting with substrates and reagents. These unique properties of nanoparticles as catalysts derive from the large percentage of coordinatively unsaturated atoms located at the surface, edges and corners of the nanoparticles compared to the total number of atoms [59]. The large surface-to-volume ratio of nanoparticles (a very high superficial area) has a straightforward positive effect on reaction rate. Besides, from industrial point of view, the advantages of make nanocatalysts an excellent catalyst is making the process more sustainable by reducing the amount of catalyst per $\mathrm{kg}$ of product [60]. Nanoparticles from metals constitutes became an important branch of catalysis in a number of industrial applications owing to their high activity and selectivity, for example, Palladium nanoparticles are employed as catalyst for the hydrogenations of unsaturated olefins owing to the ability to dissociate molecular hydrogen into the atomic state [61].

Numerous numbers of researches documented the biosynthesis and the catalytic characteristics of different nanoparticles. For example, Platinum nanoparticles obtained using Ocimum sanctum (Holy basil) extracts were shown to possess a catalytic activity to be used in the production of hydrogen fuel elements [62]. Pd nanoparticles biosynthesized using bacteria have effective performance as catalyst in dehalogenation of environmental contaminants [63]. Green gold nanoparticals showed efficient catalytic activity for the reduction of pnitrophenol to $\mathrm{p}$-aminophenol in the presence of sodium borohydride [64]. Bio fabricated silver nanoparticles showed catalytic efficiency in reduction/degradation of different organic dyes [65].

The main drawback of nanoparticles is their tendency to undergo agglomeration as they increase in size, thereby reducing the energy associated with large surface area. One route to solve this problem is by adsorbing these nanoparticles on large surface area of insoluble solids. The strong interactions between the nanoparticles and the solid support are able to adsorb nanoparticles on their surface, stabilizing them against sintering and growth. The supports have vital role in providing acidity/basicity or by tuning the electronic density of the nanoparticles [59]. Supported metal nanoparticles (Pt nanoparticles in mesoporous silica) with high catalytic performances have been applied as catalysts for biomass conversion. The bifunctional catalysis of acid and metal plays the key role for the hydrolysis and reduction of cellulose [66]. A photocatalyst of $\mathrm{TiO}_{2}$ on maghemite/silica nanocomposites has shown excellent photocatalytic performance in the selective oxidation of benzyl alcohol to benzaldehyde [67]. Figure 6 illustrates the synthesis route and application of $\mathrm{Cu}$ nanocatalyst on $\mathrm{Fe}_{3} \mathrm{O}_{4} @$ Nico magnetic nanoparticles for hydrogenation of azo dyes.

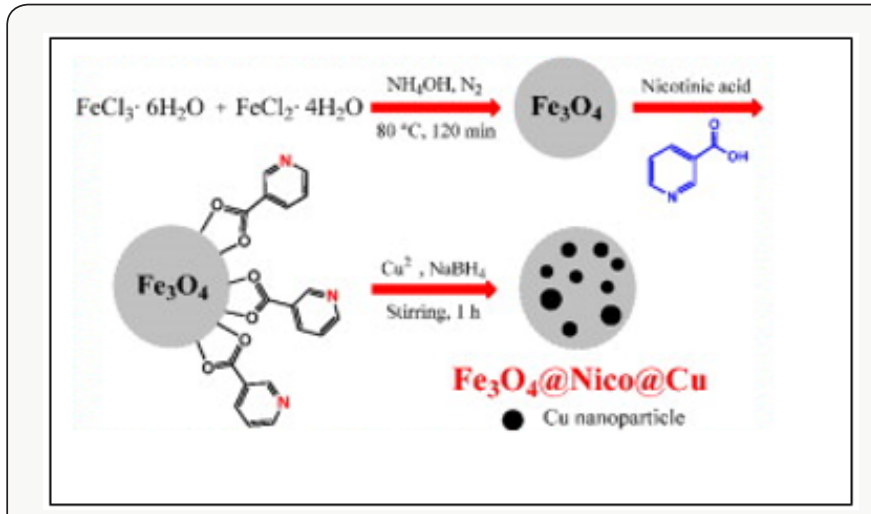

Figure 6: $\mathrm{Cu}$ nano catalyst on $\mathrm{Fe}_{3} \mathrm{O}_{4} @$ Nico magnetic nanoparticles for hydrogenation of azo dyes [68].

Photocatalysts with $\mathrm{TiO}_{2}$ coated on magnetic particles have been reported by many researchers. A photocatalyst of $\mathrm{TiO}_{2}$ on maghemite/silica nanocomposites has shown excellent photocatalytic performance in the selective oxidation of benzyl alcohol to benzaldehyde [68]. The application of natural waste materials in the field of catalysis has become a substantial research area. Recently, a number of new green strategies have been described in literature for using biosynthesis routes for catalysts production. They are based on the capacity of biomolecules to induce the formation of these catalysts. Catalysts are prepared using diverse waste treatment processes, some highly complex while others are simple to extract and isolate the desired components, which are inert/functional supports and/or active metals or metal oxides. The synthetic routes to effective catalysts can employ either a waste material alone, or their combination with additional commercial components [69]. To date, the majority of catalysts are either transition metal oxides from industrial waste or carbonaceous and alkaline earth oxides from biomass waste. A bimetallic heterogeneous nanocatalyst for [3 + 2] cycloaddition reaction with easy recovery by using an external magnetic field, was manufactured based on Fe and Pd nanoparticles [70]. Further biosynthesized heterogeneous nanocatalysts are reviwed by other researchers [60]. The key applications of biosynthesized metallic nanoparticles including catalytic applications such as reduction of environmental contaminants are well reviewed elsewhere [71].

\section{Nanomaterials Limitations and Future Prospects}

In spite of nanomaterials present unique chemical and physical properties with a wide range of applications, however, the full realization of these materials, their potential impact and industrialization is hindered by several challenging issues that are yet to be completely understood and investigated. The development of fabrication processes is essential to the industrialization of 
novel nanomaterials with lower price, and better functionality and manufacturability. The lack of a manufacturing technology capable of their production in a continuous and reproducible manner in a large scale is a big challenge. Exploring synthetic routes to largescale production with low cost is very important for the wide spread promotion of the new nanotechnology [72]. On another hand, although there has been considerable work to advance nanomaterials and its applications, understanding the occupational, health and safety aspects of engineered nanomaterials is still in its formative stage [73]. The systemic evaluation on the toxicity and environmental risks of nanomaterials is essential.

Since the properties of nanomaterials change with time and the surrounding environment, therefore, the development of nanometrology and further advancement in the existing measurement techniques and designing of new ones is required; nanoscale structures cannot be manufactured unless appropriate characterization methodologies are used [74]. One of the main challenges facing the industrialization of nanomaterials for catalysis is the lack of in-situ information about catalysts. This factor has limited the understanding of catalytic mechanisms and the development of catalysts with high selectivity and activity [75]. Selectivity is a critically important issue for the commercialization of nano catalyst; achieving $100 \%$ selectivity to minimize the formation of byproducts is a crucial challenge in catalysis that is not fully addressed. Cost and time effective technologies for recovery of nanocatalysts and nanoadsorbents used in industrial processes is another critical issue needs more researches and investigations. Increasing the sensor sensitivity (lowering the detection limits), the operational lifetime and cost are also among the challenging issues face the industrialization of nano sensors.

Reproducibility, reliability, and stability of devices based on nanomaterials are the main factors need to be considered. Researchers must keep in mind that when dealing with complex or hybrid structures, stability may be reduced, therefore, it is important to study the device as a whole, not just the nanomaterial. Furthermore, a general challenge faces the manufacturing technologies of nanomaterials is the using capping ligands. The capping ligands can potentially interfere with the final applications of the nanomaterials in microdevices. The development of novel manufacturing technologies must be addressed in the further research for the continuous synthesis of nanomaterials with tailored compositions and tunable sizes in the absence of capping ligands.

\section{Conclusion}

Wide spectrum of nanomaterials has created huge interest among the scientific community in recent years. This immense interest is due to their unique properties, mainly arising from the quantum confinement effect and extremely large surface-tovolume ratios. Different types of nanomaterials; monodispersed and composites based on metals, metal oxides, metal alloys, carbon, and polymers have been manufactured and already are being commercialized and used in various applications including thin layer coatings, sensing, (drug, gene, protein and vaccine) delivery, as well as adsorption and catalysis. However, the full realization of these nanomaterials, their potential impact and industrialization is hindered by several challenging issues that are yet to be completely understood and investigated.

\section{References}

1. Charitidis CA, Georgiou P, Koklioti MA, Trompeta AF, Markakis V (2014) Manufacturing nanomaterials: from research to industry. Manufacturing Rev 1: 11 .

2. Ko WY, Jing-Zhi Chen, Yin Cheng Yan, Kuan-Jiuh Lin (2012) International Conference and Exhibition on Nanotechnology \& Nanomedicine, March 12-14, Omaha Marriott, USA.

3. Schulte J (2005) Nanotechnology: Global Strategies, Industry Trends and Applications.

4. Park J, An K, Hwang Y, Park JG, Noh HJ, et al. (2004) Ultra-large-scale syntheses of monodisperse nanocrystals. Nature materials 3: 891-895.

5. Pilotek S, Tabellion F (2005) Nanoparticles in coatings. European Coatings Journal 4: 170.

6. Jiang CC, Cao YK, Xiao GY, Zhuab RF, Lu YP (2017) A review on the application of inorganic nanoparticles in chemical surface coatings on metallic substrates. RSC 7: 7531-7539.

7. Enoki T, Kiguchi M (2018) Challenges for single molecule electronic devices with nanographene and organic molecules. Physica Scripta 93(5): 53001.

8. Engberg S, Agersted K, Crovetto A, Hansen O, Lame YM, et al. (2017) Investigation of Cu2ZnSnS4 nanoparticles for thin-film solar cell applications. Thin Solid Films 628(30): 163-169.

9. Vidor FF, Meyers T, Hilleringmann U (2016) Inverter Circuits Using ZnO Nanoparticle Based Thin-Film Transistors for Flexible Electronic Applications. Nanomaterials 6(9): 154.

10. Hao J, Wang QK, Weimer W, Abell J, Wilson M (2015) SERS Spectra of Permethrin on Silver Nanofilm. American Journal of Nano Research and Applications 3(1-1): 29-32.

11. Haider AJ, AL Anbari RH, Kadhim GR, Salame CT (2017) Exploring potential Environmental applications of TiO2 Nanoparticles. Energy Procedia 119: 332-345.

12. Mora LV, Naik S, Paul S, Dawson R, Neville A, et al. (2017) Influence of silica nanoparticles on corrosion resistance of sol-gel based coatings on mild steel. Surface and Coatings Technology 324(15): 368-375.

13. Manoudis P, Papadopoulou S, Karapanagiotis I, Tsakalof A, Zuburtikudis I, et al. (2007) Polymer-Silica nanoparticles composite films as protective coatings for stone-based monuments. J. Phys.: Conf. Ser 61: 1361-1365.

14. Juknius T, Ružauskas M, Tamulevičius T, Šiugždinienė R, Juknienė I, et al. (2016) Antimicrobial Properties of Diamond-Like Carbon/Silver Nanocomposite Thin Films Deposited on Textiles: Towards Smart Bandages. Materials 9(5): 371.

15. Al Aani S, Haroutounian A, Wright CJ, Hilal N (2018) Thin Film Nanocomposite (TFN) membranes modified with polydopamine coated metals/carbon-nanostructures for desalination applications. Desalination, 427: 60-74.

16. Singh RS (2018) Thermal-induced SPR tuning of Ag-ZnO nanocomposite thin film for plasmonic applications S.K. Applied Surface Science 439: 919-926. 
17. Dahman Y (2017) In Nanotechnology and Functional Materials for Engineers. Micro and Nano Technologies, Chapter 4 - Nanosensors p. 67-91.

18. Hu Q, Wujcik EK, Kelarakis A, Cyriac J, Gong X (2017) Carbon-Based Nanomaterials as Novel Nanosensors. Journal of Nanomaterials 2017: $1-2$.

19. Doria G, Conde J, Veigas B, Giestas L, Almeida C, et al. (2012) Noble Metal Nanoparticles for Biosensing Applications. Sensors (Basel) 12(2): 16571687.

20. Priyadarshini E, Pradhan N (2017) Gold nanoparticles as efficient sensors in colorimetric detection of toxic metal ions: A review. Sensors and Actuators B: Chemical 238: 888-902.

21. Vellaichamya B, Periakaruppan P (2017) Size and shape regulated synthesis of silver nanocapsules for highly selective and sensitive ultralow bivalent copper ion sensor application. New Journal of Chemistry, 41(10): 4006-4013.

22. Qiong Z, Jin SC, Xiao FL, Hao TB, Jian HJ (2011) Palladium nanaparticles/ chitosan-grafted grapheme nanocomposites for construction of a glucose biosensor. Biosens. Bioelectron 26(8): 3456-3463.

23. Rackauskas S, Barolo C (2017) ZnO Nanowire Application in Chemoresistive Sensing: A Review. Nanomaterials 7(11): 381.

24. Cui Y, Wei Q Park H, Lieber CM (2001) Nanowire Nanosensors for Highly Sensitive and Selective Detection of Biological and Chemical Species. Science 293(5533): 1289-1292.

25. Comin E (2016) Metal oxide nanowire chemical sensors: innovation and quality of life. Materials today, 19(10): 559-567.

26. Luo J, Wu Y, Lin S (2015) Review: Recent Developments of Carbon Nanotubes Hybrid Assemblies for Sensing, American Journal of Nano Research and Applications. Special Issue: Nanomaterials and Nanosensors for Chemical and Biological Detection 3(1-1): 23-28.

27. Khan S, Cao Q Zheng YM, Huang YZ, Zhu YG (2008) Health risks of heavy metals in contaminated soils and food crops irrigated with wastewater in Beijing, China. Environ. Pollut 152(3): 686-692.

28. Gupta VK, Tyagi I, Sadegh H, Shahryari-Ghoshekandi R, Makhlouf AH, et al. (2015) Nanoparticles as Adsorbent; A Positive Approach for Removal of Noxious Metal Ions: A Review. Sci. Technol. Dev 34(3): 195-214.

29. Ali I (2012) New Generation Adsorbents for Water Treatment. Chem. Rev 112(10): 5073-5091.

30. Taman R, Ossman ME, Mansour MS, Farag HA (2015) Metal Oxide Nanoparticles as an Adsorbent for Removal of Heavy Metals. J Adv Chem Eng 5: 125 .

31. Chiavola A, Amato E, Stoller M, Chianese A, Boni MR (2016) Application of iron-based nanoparticles as adsorbents for arsenic removal from water, Chemical Engineering Transactions 47: 325-330.

32. Iconaru SL, Guégan R, Popa CL, Motelica Heino M, Ciobanu CS, et al. (2016) Magnetite (Fe304) nanoparticles as adsorbents for $\mathrm{As}$ and $\mathrm{Cu}$ removal. Applied Clay Science 134 Part 2: 128-135.

33. Ul Islam M, Ullah MW Khan S, Manan S, Khattak W, Ahmad W, et al. (2017) Current advancements of magnetic nanoparticles in adsorption and degradation of organic pollutants. Environ Sci Pollut Res Int 24(14) : 12713-12722.

34. Tadjarodi A, Imania M, Salehi M (2015) ZnFe2O4 nanoparticles and a clay encapsulated $\mathrm{ZnFe204}$ nanocomposite: synthesis strategy, structural characteristics and the adsorption of dye pollutants in water. RSC 5(69): 56145-56156.

35. Ravindhranath K, Ramamoorty M (2016) Nickel Based Nano Particles as Adsorbents in Water Purification Methods - A Review. Oriantal Journal of Chemistry 33(4): 1603-1613.

36. Basheer A (2018) New generation nano-adsorbents for the removal of emerging contaminants in water. Journal of Molecular Liquids 261(1): 583-593.

37. Guerra FD, Attia MF, Whitehead DC, Alexis F (2018) Review: Nanotechnology for Environmental Remediation: Materials and Applications. Molecules 23(7): 1-23.

38. Khaleel A, Kapoor PN, Klabunde KJ (1999) Nanocrystalline Metal Oxides as New Adsorbents for Air Purification. Nanostructured Materials 11(4): 459-468.

39. Alonso A, Moral Vico J, Abo Markeb A, Busquets Fité M, Komilis D, Puntes V, et al. (2017) Critical review of existing nanomaterial adsorbents to capture carbon dioxide and methane. Sci Total Environ 595: 51-62.

40. Hosseini A, Hassankiadeh MN (2017) Parametric study of sweetening process of sour gas by Molybdenum oxide nanoparticles. Braz J Chem Eng 34(4): 1191-1202.

41. Babu DJ, Puthusseri D, Kühl FG Okeil1 S, Bruns M, Hampe M, et al. (2018) SO2 gas adsorption on carbon nanomaterials: a comparative study. Nanotechnol 9: 1782-1792.

42. Mohamed EF (2017) Nanotechnology: Future of Environmental Air Pollution Control. Environmental Management and Sustainable Development 6(2): 429-455.

43. De Jong WH, Borm PJA (2008) Drug delivery and nanoparticles: Applications and hazards. Int J Nanomedicine 3(2): 133-149.

44. Yu X, Trase I, Ren M, Duval K, Guo X, et al. (2016) Review Article: Design of Nanoparticle-Based Carriers for Targeted Drug Delivery. Journal of Nanomaterials p. 1-15.

45. Wilczewska AZ, Niemirowicz K, Markiewicz KH, Car H (2012) Nanoparticles as drug delivery systems. Pharmacological Reports 64 (5): 1020-1037.

46. Kumar B, Jalodia K, Kumar P, Gautam HK (2017) Recent advances in nanoparticle-mediated drug delivery. Journal of Drug Delivery Science and Technology 41:260-268.

47. Kudr J, Haddad Y, Richtera L, Heger Z, Cernak M, et al. (2017) Magnetic Nanoparticles: From Design and Synthesis to Real World Applications. Nanomaterials 7(9): 243.

48. Herranz F, Almarza E, Rodríguez I, Salinas B, Rosell Y, et al. (2011) The Application of Nanoparticles in Gene Therapy and Magnetic Resonance Imaging. Microsc Res Tech 74(7): 577-591.

49. Riley MK, Vermerris W (2017) Recent Advances in Nanomaterials for Gene Delivery-A Review. Nanomaterials (Basel) 7(5): 94.

50. Blanco JLJ, Benito JM, Mellet CO, Fernández JMG (2017) Molecular nanoparticle-based gene delivery systems. Journal of Drug Delivery Science and Technology 42: 18-37.

51.A. Werfe T, Duvall C (2016) Polymeric nanoparticles for gene delivery. pp.147-188. In: Ravin Narain Polymers and Nanomaterials for Gene Therapy.

B. Mukhopadhyay P, Mishra R, Rana D, Kundua PP (2012) Strategies for effective oral insulin delivery with modified chitosan nanoparticles: A review. Progress in Polymer Science 37(11): 1457-1475.

52. Sharma G, Sharma AR, Nam JS, Doss GPC, Lee SS, etal.(2015) Nanoparticle based insulin delivery system: the next generation efficient therapy for Type 1 diabetes. J Nanobiotechnology 13: 74

53. Liu Y, Chen C (2016) Role of nanotechnology in HIV/AIDS vaccine development. Adv Drug Deliv Rev 103: 76-89.

54. Kong FY, Zhang JW, Li RF, Wang ZX, Wang WJ, et al. (2017) Unique Roles of Gold Nanoparticles in Drug Delivery, Targeting and Imaging Applications. Molecules 22(9): 1445.

55. Chaturvedi S, Dave PN, Shah NK (2012) Applications of nano-catalyst in new era. Journal of Saudi Chemical Society 16(3): 307-325. 
56. Singh SB, Tandon PK (2014) Catalysis: A Brief Review on Nano-Catalyst. Journal of Energy and Chemical Engineering 2(3): 106-115.

57. Schröfel A, Kratošová G (2011) Biosynthesis of Metallic Nanoparticles and Their Applications. From A Prokop (ed.) Intracellular Delivery: Fundamentals and Applications, Fundamental Biomedical Technologies 5: 373-409.

58. Crooks RM, Zhao M, Sun LI, Chechik V, Yeung LK (2001) DendrimerEncapsulated Metal Nanoparticles: Synthesis, Characterization, and Applications to Catalysis. 34(3): 181-190.

59. Navalón S, García H (2016) Nanoparticles for Catalysis. Nanomaterials (Basel) 6(7): 123.

60. Palomo JM, Filice M (2016) Biosynthesis of Metal Nanoparticles: Nove Efficient Heterogeneous Nanocatalysts. Nanomaterials 6(5): 84.

61. Saldan I, Semenyuk Y, Marchuk I, Reshetnyak O (2015) Chemical synthesis and application of palladium nanoparticles. J Mater Sci 50(6): 2337-2354.

62. Soundarrajan C, Sankari A, Dhandapani P, Maruthamuthu S, Ravichandran S, et al. (2012) Rapid biological synthesis of platinum nanoparticles using Ocimum sanctum for water electrolysis applications. Bioprocess Biosyst Eng 35(5): 827-833.

63. Hennebel T, De Corte S, Verstraete W, Boon N (2012) Microbial production and environmental applications of $\mathrm{Pd}$ nanoparticles for treatment of halogenated compounds. Curr Opin Biotechnol 23(4): 555561.

64. Kherde YA (2014) Green Synthesis and Evaluation of Catalytic Activity of Sugar Capped Gold Nanoparticles. Masters Theses \& Specialist Projects. Western Kentucky University, Bowling Green, Kentucky, USA.

65. Bogireddy NKR, Kumar HAK, Mandal BK (2016) Biofabricated silver nanoparticles as green catalyst in the degradation of different textile dyes. Journal of Environmental Chemical Engineering 4(1): 56-64.
66. Fukuoka A, Dhepe PL (2009) Sustainable green catalysis by supported metal nanoparticles. Chem Rec 9(4): 224-235.

67. Colmenares JC, Ouyang W, Ojeda M, Kuna E, Chernyayeva O, et al. (2015) Mild ultrasound-assisted synthesis of $\mathrm{TiO} 2$ supported on magnetic nanocomposites for selective photo-oxidation of benzyl alcohol. Appl Catal B: Environmental 183: 107-112.

68. Amir Md, Kurtan U, Baykal A (2015) Synthesis and application of magnetically recyclable nanocatalystFe304@Nico@Cu in the reduction of azo dyes. Chinese Journal of Catalysis 36(8): 1280-1286.

69. Bennett JA, Wilson K, Lee AF (2016) Catalytic applications of waste derived materials. J Mater Chem A 4(10): 3617-3637.

70. Mishra K, Basavegowda N, Lee YR (2015) Biosynthesis of Fe, Pd, and Fe$\mathrm{Pd}$ bimetallic nanoparticles and their application as recyclable catalysts for $[3+2]$ cycloaddition reaction: A comparative approach. Catal Sci Technol 5: 2612-2621.

71. Schröfel A, Kratošová G, Šafařík, Šafaříková M, Raška I, et al. (2014) Applications of biosynthesized metallic nanoparticles - a review. Acta Biomater 10(10): 4023-4042.

72. Zang F (2017) Grand Challenges for Nanoscience and Nanotechnology in Energy and Health. Front Chem 5: 80.

73. Yokel RA, MacPhail RC (2011) Engineered nanomaterials: exposures, hazards, and risk prevention. Occup Med Toxicol 6: 7.

74. Linkov P, Artemyev M, Efimovcd AE, Nabiev I (2013) Comparative advantages and limitations of the basic metrology methods applied to the characterization of nanomaterials. Nanoscale 5(19): 8781-8798.

75. Zhang S, Nguyen L, Zhu Y, Zhan S, Tsung CK, et al. (2013) In-situ studies of nanocatalysis. Acc Chem Res 46(8): 1731-1739.

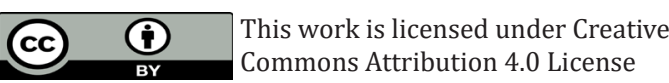

To Submit Your Article Click Here: Submit Article

DOI: $10.32474 /$ OAJBEB.2018.02.000149

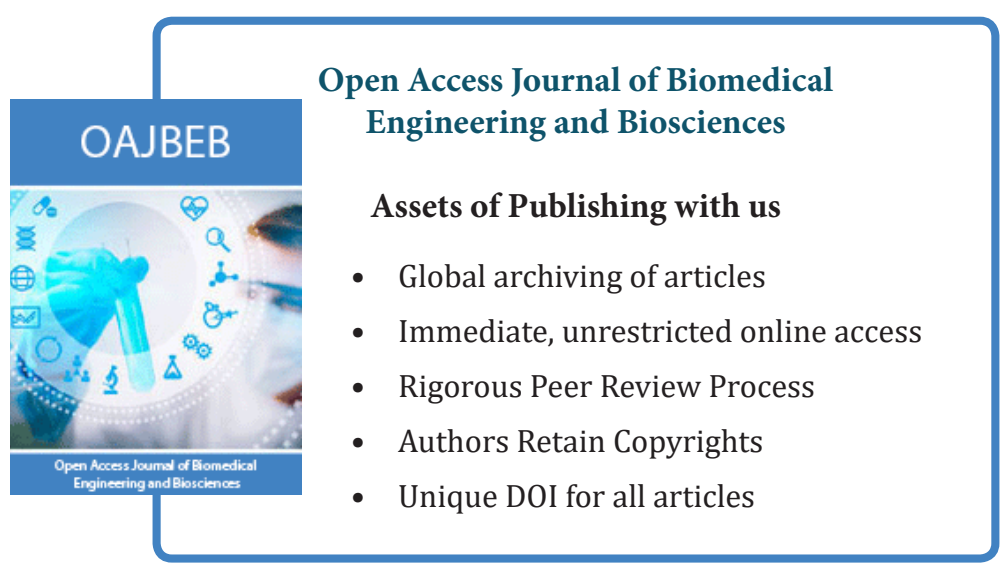

